\title{
Solo Reduced Port Laparoscopic Left Lateral Sectionectomy
}

\author{
YoungRok Choi, M.D., Ho-Seong Han, M.D., Ph.D., Yoo-Seok Yoon, M.D., Ph.D., Jai Young Cho, M.D., Ph.D., Sungho Kim, M.D., \\ In Gun Hyun, M.D., Kil Hwan Kim, M.D. \\ Department of Surgery, Seoul National University Bundang Hospital, Seoul National University College of Medicine, Seongnam, Korea
}

\begin{abstract}
Laparoscopic approach for left lateral sectionectomy became the standard procedure. With increasing demand for minimizing of the access ports and with the advancement in surgical technique, reduced port laparoscopic surgery is introducing itself to the fields of hepatic surgery. We report a case of solo reduced port laparoscopic left lateral sectionectomy for a 25-year-old patient with a $1.7 \mathrm{~cm}$ sized tumor implant of growing teratoma syndrome. She underwent salpingo-oophorectomy and 3 cycles of chemotherapy with bleomycin, etoposide and cisplatin 2 prior to the operation. Her BMI was 18.3 $\mathrm{kg} / \mathrm{m}^{2}$. The total operation time was 85 minutes and estimated blood loss was scanty. The patient was discharged without a significant complication on postoperative day 5. In the video, we demonstrate the surgical procedure of the solo reduced port laparoscopic left lateral sectionectomy using a laparoscopic scope holder.
\end{abstract}

Keywords: Laparoscopic surgery, Hepatectomy, Minimally invasive surgical procedures

Supplementary video file: This article contains supplementary material (https://doi.org/10.7602/ jmis.2018.21.3.133).

This is an Open Access article distributed under the terms of the Creative Commons Attribution Non-Commercial License (http:// creativecommons.org/licenses/by-nc/4.0/) which permits unrestricted non-commercial use, distribution, and reproduction in any medium, provided the original work is properly cited.
Received July 28, 2017

Accepted August 16, 2017

Corresponding author

Ho-Seong Han

Department of Surgery, Seoul

National University Bundang

Hospital, 82 Gumi-ro, 173 beon-

gil, Bundang-gu, Seongnam 13620,

Korea

Tel: +82-31-787-7099

Fax: +82-31-787-4078

E-mail: hanhs@snubh.org

ORCID:

http://orcid.org/0000-0001-9659-1260

Copyright $\odot 2018$ The Journal of Minimally Invasive Surgery. All rights reserved.

\section{INTRODUCTION}

Laparoscopic liver surgery is attracting wider interest for the treatment of benign and malignant neoplasms. ${ }^{1,2}$ Relative to open resection, it offers decreased length of hospital stay, less blood loss, less postoperative pain, faster recovery, better cosmesis, and lower morbidity and mortality. ${ }^{3}$ Oncological outcomes are comparable to those of open liver resection for HCC. ${ }^{2,45}$ Moreover, reduced port surgery has been attracting attention in the field of laparoscopic hepatectomy with much less surgical invasiveness. However, it is not easy to apply the reduced port hepatectomy because of technical difficulties and unstable surgical view. We report our experience of reduced port laparoscopic left lateral sectionectomy without a scopist.

\section{PATIENT AND SURGICAL PROCEDURE}

A 25 years old patient underwent salpingo-oophorectomy and chemotherapy (bleomycin, etoposide and cisplatin) 3 times because of $15 \mathrm{~cm}$ sized immature teratoma and finished her treatment two years ago. Recently hepatic mass was detected in liver segment 2 and she ws diagnosed with growing teratoma syndrome.

The patient was put in supine position with the legs split apart. The operator was between patients' legs during the operation. An $11 \mathrm{~mm}$ trans-umbilical port for a camera, two 12 $\mathrm{mm}$ working ports in the both subcostal area, were inserted. Another $2 \mathrm{~mm}$ sized incision was made for applying a Pringle's maneuver. A 10 mm flexible laparoscopic videoscope (Endoeye flex, Olympus medical systems corp., Tokyo, Japan) was 


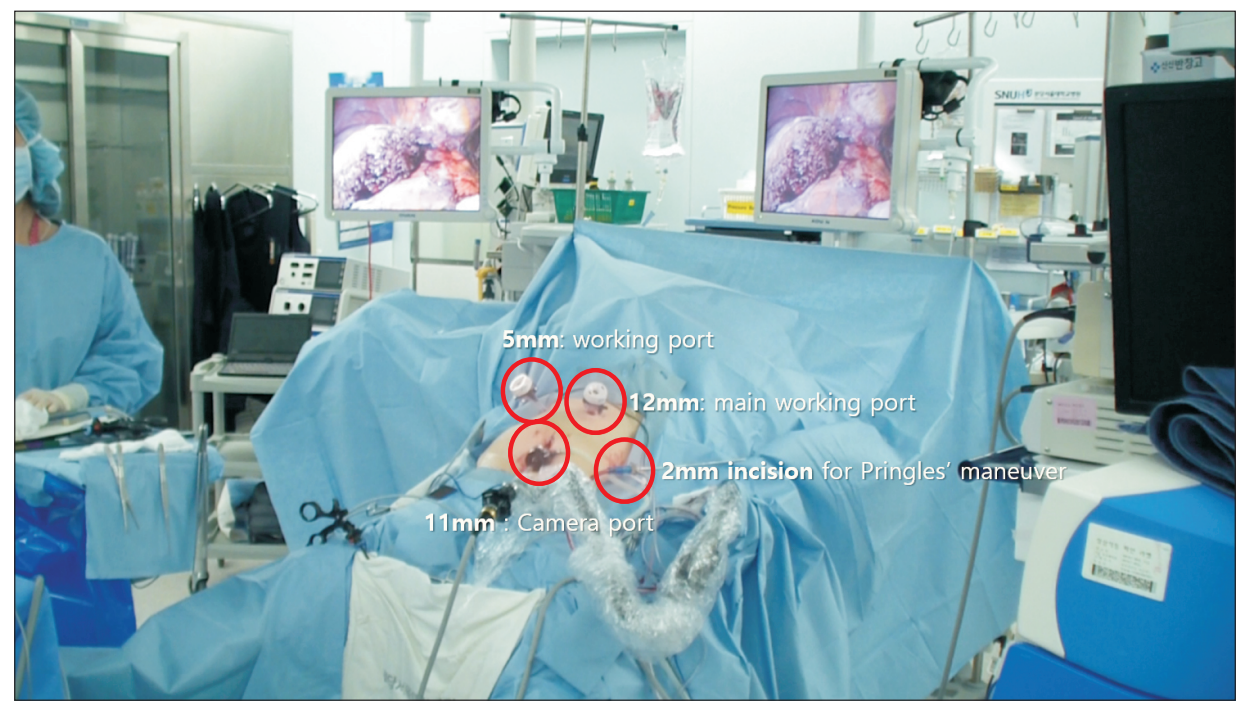

Fig. 1. Surgical field in solo reduced port laparoscopic hepatectomy. A $10 \mathrm{~mm}$ laparoscopic flexible camera was fixed using a laparoscopic scope holder which was installed in the left rail of the bed. The camera was inserted through the 11 $\mathrm{mm}$ port at the umbilicus. $12 \mathrm{~mm}$ and $5 \mathrm{~mm}$ ports were inserted in the both subcostal area. Another $2 \mathrm{~mm}$ incision for application of Pringles' maneuver was made.

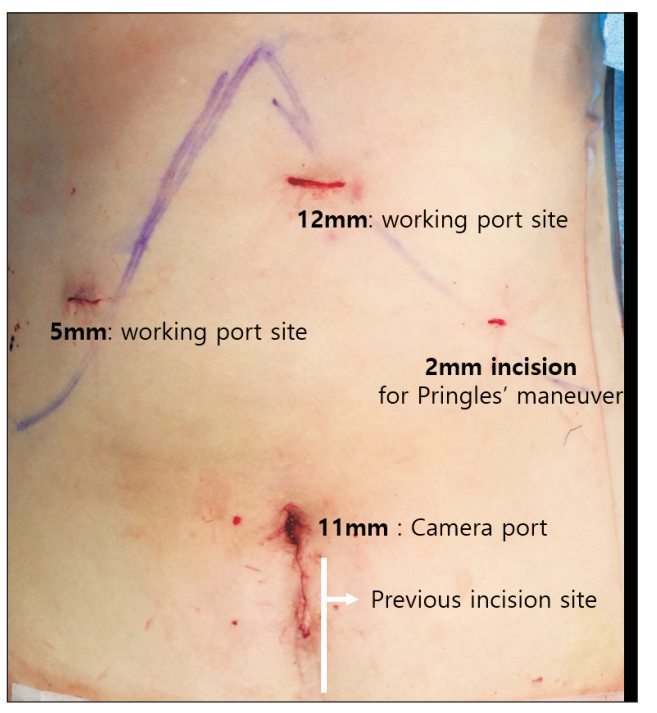

Fig. 2. Surgical wound. The specimen was extracted the umbilical port site with extension along the previous incision site.

fixed using a laparoscopic scope holder (Laparostat ${ }^{\mathrm{TM}}$, CIVCO medical Solutions, IA, USA) (Fig. 1). Superficial parenchymal dissection was done with an energy device. After encircling the hepatodeuodenal ligament through the foramen of Winslow with a nylon tape for Pringle's maneuver, deep part of the liver was dissected with a cavitron ultrasonic surgical aspirator (CUSA).

Several small Glissonean pedicles to S3 were resected, and a large Glissonean pedicle to S2 was isolated and resected. The left hepatic vein was resected at the conjoint level bewteen middle hepatic and left hepatic vein using an automatic stapler (iDrive $^{\mathrm{TM}}$, Medtronic, MN, USA). The specimen was removed through the umbilical incision site using a vinyl bag (Fig. 2).
All surgical procedures were performed by one operator with help of a scrub nurse only.

\section{RESULTS}

The total operation time was 85 minutes and estimated blood loss was scanty. The patient was discharged without significant complication on postoperative day 5 .

\section{DISCUSSION}

Laparoscopic hepatectomy for the left sided hepatic lesion is a safe and effective approach in the hands of well-organized surgical team including a surgeon and a scopist. Laparoscopic approach for left lateral sectionectomy has already become the standard procedure. ${ }^{6}$ As the emphasis on minimizing the access ports with the current advancements in surgical techniques, reduced port laparoscopic surgery is introducing itself in the field of liver resection. ${ }^{7.8}$

However, it is difficult to make a stable surgical view to perform operation especially in the reduced port hepatectomy without or with minimal assistance even in the left lateral sectionectomy. When an inexperienced scopist joins the laparoscopic hepatectomy, the operation becomes more difficult. In the era where there is a shortage of trained human resources, an inexperienced scopist might be a big barrier to perform a reduced port laparoscopic hepatectomy.

Therefore, we introduced the solo laparoscopic left lateral sectionectomy using an unexhausted scope holder under the reduced ports in this video. Although a scope holder cannot totally replace a scopist, it might make a steady surgical view in limited surgical cases. In laparoscopic left lateral sectionectomy, the resection plane is parallel to the camera axis 
from the umbilical port. Therefore, the camera can show the surgical field with minimal movement of the scope during the operation. This solo procedure combined with a reduced port hepatectomy enables the operation without a scopist or to an extent, it makes it easier than the operation with an inexperienced scopist.

In conclusion, this solo surgery can be one of the options to make a successful reduced port laparoscopic hepatectomy with a stable surgical view in limited cases.

\section{ACKNOWLEDGMENTS}

Authors thank Woo Chul Kim, M.D. for your help for improving the use of English in the manuscript.

\section{REFERENCES}

1) Aldrighetti L, Guzzetti E, Ferla G. Laparoscopic hepatic left lateral sectionectomy using the LaparoEndoscopic Single Site approach: evolution of minimally invasive liver surgery. J Hepatobiliary Pancreat Sci 2011;18:103-105.

2) Guro H, Cho JY, Han HS, Yoon YS, Choi Y, Periyasamy M. Cur- rent status of laparoscopic liver resection for hepatocellular carcinoma. Clin Mol Hepatol 2016;22:212-218.

3) Ban D, Kudo A, Irie T, et al. Advances in reduced port laparoscopic liver resection. Asian J Endosc Surg 2015;8:11-15.

4) Han HS, Shehta A, Ahn S, Yoon YS, Cho JY, Choi Y. Laparoscopic versus open liver resection for hepatocellular carcinoma: Case-matched study with propensity score matching. J Hepatol 2015;63:643-650.

5) Takahara T, Wakabayashi G, Beppu T, et al. Long-term and perioperative outcomes of laparoscopic versus open liver resection for hepatocellular carcinoma with propensity score matching: a multi-institutional Japanese study. J Hepatobiliary Pancreat Sci 2015;22:721-727.

6) Wakabayashi G, Cherqui D, Geller DA, et al. Recommendations for laparoscopic liver resection: a report from the second international consensus conference held in Morioka. Ann Surg 2015;261:619-629.

7) Tayar C, Subar D, Salloum C, Malek A, Laurent A, Azoulay D. Single incision laparoscopic hepatectomy: Advances in laparoscopic liver surgery. J Minim Access Surg 2014;10:14-17.

8) Gkegkes ID, Iavazzo C. Single incision laparoscopic hepatectomy: A systematic review. J Minim Access Surg 2014;10:107-112. 